\title{
A Systems Approach and Skeletal Myogenesis
}

\author{
Yoshiaki Ito,, ${ }^{1,2}$ Tomohiro Kayama, ${ }^{1,3}$ and Hiroshi Asahara ${ }^{1,2}$ \\ ${ }^{1}$ Department of Systems BioMedicine, Graduate School of Medical and Dental Sciences, Tokyo Medical and Dental University, \\ Bunkyo, Tokyo 113-8510, Japan \\ ${ }^{2}$ Department of Systems BioMedicine, National Research Institute for Child Health and Development, Setagaya, \\ Tokyo 157-8535, Japan \\ ${ }^{3}$ Department of Orthopaedic Surgery, The Jikei University School of Medicine, Minatoku, Tokyo 105-8461, Japan
}

Correspondence should be addressed to Hiroshi Asahara, asahara.syst@tmd.ac.jp

Received 21 March 2012; Revised 27 May 2012; Accepted 28 June 2012

Academic Editor: Daniela Palacios

Copyright ( 2012 Yoshiaki Ito et al. This is an open access article distributed under the Creative Commons Attribution License, which permits unrestricted use, distribution, and reproduction in any medium, provided the original work is properly cited.

\begin{abstract}
Skeletal myogenesis depends on the strict regulation of the expression of various gene subsets. Therefore, the understanding of genome wide gene regulation is imperative for elucidation of skeletal myogenesis. In recent years, systems approach has contributed to the understanding of various biological processes. Our group recently revealed the critical genome network of skeletal myogenesis by using a novel systems approach combined with whole-mount in situ hybridization (WISH) database, highthroughput screening, and microarray analysis. In this paper, we introduce our systems approach for understanding the myogenesis regulatory network and describe the advantages of systems approach.
\end{abstract}

\section{Introduction}

Skeletal muscle is indispensable for any moving function of the human body, and abnormality of the skeletal muscle causes great disability in affected people. It is therefore important to understand the mechanism of skeletal myogenesis so that it may form a basis for disease treatment.

Almost all skeletal muscles in the body derive from dermomyotome or myotome in somites. The myotome and dermomyotome contain myogenic progenitor cells that evolve into skeletal muscles, aggregates of myofibers, in the whole body. During skeletal myogenesis, myofibers form from myogenic progenitors, where distinct subsets of genes are activated or repressed and form a complex molecular network of interdependent pathways [1-3]. These processes are mainly regulated by the muscle-specific basic helix-loophelix (bHLH) transcription factors, MyoD, Myf5, Myogenin (Myog), and Mrf4. Analysis of null mice of these genes suggested that MyoD and Myf5 play a role in determining the myogenic progenitors to myoblasts [4]. Myog is important in differentiation from myoblasts to myotubes $[5,6]$, and Mrf4 is important in both determination and differentiation [7]. The described transcription factors are class II (tissue-specific) bHLH transcription factors capable of either homodimerization or heterodimerization with class I bHLH factors, such as E-proteins HEB/HTF4, E2-2/ITF2, and E12/E47 [8]. All bHLH dimers bind to an E-box, a consensus sequence comprised of the sequence CANNTG. Id proteins have been identified to act as myogenic antagonists by directly binding to E-proteins and/or muscle-specific bHLH proteins, blocking their ability to bind E-boxes and activate transcription at muscle-specific promoters [9-11]. IdmRNAs are detected in proliferating skeletal muscles and are downregulated in differentiated muscle cultures $[9,12]$. This downregulation was thought to be important for skeletal muscle formation; however, the mechanism of Id repression has not been understood for almost 20 years.

Recently, we revealed the Id downregulation mechanism in myogenesis by our own systems approach combined with WISH database, high-throughput screening, and microarray analysis [13]. Systems approach, a systematic study using various comprehensive analyses such as high-throughput sequencing technologies, genome wide cell-based assays, and bioinformatics, has allowed us to expand our knowledge of life phenomenon. We have reviewed studies that have utilized systems approach. In addition to this, we describe our own 
systems approach and how this has helped in understanding skeletal myogenesis.

\section{Deep Sequencing and Array-Based Approaches}

High-throughput sequencing technologies allow highresolution, genome wide investigation of epigenetic conditions. For instance, mapping of open chromatin regions, histone modifications, and DNA methylation across a whole genome is now feasible, and whole transcripts including noncoding RNAs (ncRNAs) can be identified via RNA sequencing.

These high-throughput sequencing-based technologies and microarray-based ChIP chip analyses are used in various fields, and there have been reports on embryonic stem (ES) cells. Meissner et al. analyzed genome-scale DNAmethylation profiles and histone methylation patterns of mouse ES cells and differentiated cells by using highthroughput bisulphite sequencing and ChIP-sequence [14]. This revealed that DNA methylation patterns are better correlated with histone methylation patterns than with the underlying genome sequence context and that methylation of CpGs is one of dynamic epigenetic marks during differentiation particularly in regulatory regions outside of core promoters [14]. Also, Bock et al. analyzed DNA methylation patterns and gene expression of 20 human ES cell lines and 12 human iPS cell lines, identifying epigenetic and transcriptional similarity of ES and iPS cells [15]. Bernstein et al. mapped Polycomb-associated Histone H3 Lysine 27 trimethylation (H3K27me3) and Trithorax-associated Histone H3 Lysine 4 trimethylation (H3K4me3) across the whole genome in mouse ES cells by ChIP-chip analysis [16]. H3K27me3 is an epigenetic mark that mediates gene silencing, whereas H3K4me3 occurs in nucleosomes found in the promoter regions of actively transcribed genes. They identified a specific modification pattern consisting of large regions of H3K27me3 harboring smaller regions of $\mathrm{H} 3 \mathrm{~K} 4 \mathrm{me} 3$. It has been proposed that this "active" and "repressive" modification pattern represents genes specifically designed to initiate transcription, and this active state is thought to be essential for the developmental potential of ES cells. Pan et al. also mapped H3K27me3 and H3K4me3 across the whole genome in human ES cells [17]. The vast majority of H3K27me3 colocalized on genes modified with $\mathrm{H} 3 \mathrm{~K} 4 \mathrm{me} 3$ as within mouse ES cells. These commodified genes displayed low expression levels and were enriched in developmental gene function. Another significant set of genes lacked both modifications, also expressed at low levels in ES cells, but was enriched for gene function in physiological responses rather than development. Commodified genes change expression levels rapidly during differentiation, but so do a substantial number of genes in other modification categories. Pluripotency-associated genes such as SOX2, OCT4, and NANOG shifted from modification by $\mathrm{H} 3 \mathrm{~K} 4 \mathrm{me} 3$ alone to colocalization of both modifications as they were repressed during differentiation. These data revealed that $\mathrm{H} 3 \mathrm{~K} 27 \mathrm{me} 3$ modifications change during early differentiation, both relieving existing repressive domains and imparting new ones, and that colocalization with $\mathrm{H} 3 \mathrm{~K} 4 \mathrm{me} 3$ is not restricted to pluripotent cells. Highthroughput sequencing technologies are also used in the studies on genome-wide ncRNA expression analysis. Calabrese et al. analyzed short RNA expression in Dicer-positive and Dicer-knockout mouse ES cells [18]. From quantification of miRNA levels, they estimated that there are 130,000 5' phosphorylated short RNAs per ES cell. 15\% of these RNAs are generated independently of the Dicer gene, presumed breakdown products of mRNAs, which are low in abundance and consist of highly repetitive sequences. The remaining $85 \%$ of $5^{\prime}$ phosphorylated ES cell short RNAs consist of miRNAs or miRNA-like species that depend on Dicer for biogenesis. The majority of ES cell miRNAs appear to be generated by six distinct loci, four of which have been implicated in cell cycle control or oncogenesis. At a depth of sequencing that approaches the total number of $5^{\prime}$ phosphorylated short RNAs per cell, miRNAs appeared to be Dicer's only substrate. These studies identified genome-wide epigenetic marks and gene expression in ES cells. They have obtained data revealing the characteristics of ES cells and also incidentally discovered "active" and "repressive" histone comodification patterns. This had been possible due to a genome-wide analysis, thus indicating the importance of such approach.

The systems approach is also beneficial to reveal the regulatory network of skeletal myogenesis. Myogenesis is orchestrated through a series of transcriptional controls regulated by the myogenic bHLH factors. Several groups performed ChIP-chip analysis to identify targets of myogenic regulatory factors $[1,2]$. These analyses indicated overlapping of distinct targets of MyoD and Myog suggesting the mechanism of sequential expression during myogenesis. At early myogenesis, MyoD is sufficient for activation of the expression, and these genes are expressed immediately after MyoD induction. On the other hand, during late myogenesis, MyoD initiates only regional histone modification. Myog does not bind without MyoD, and the expression of late genes requires both MyoD and Myog. In recent years, genome-wide MyoD target profiling using ChIP-sequence analysis has been reported [19]. High-throughput sequencing technology-based ChIPsequence analysis suggested over 20,000 MyoD-binding sites, greater than with the array-based ChIP-chip analysis [19]. This analysis identified that MyoD was constitutively bound to thousands of sites in both myoblasts and myotubes and that the genome wide MyoD binding was related with regional histone acetylation [19]. This suggests that myogenic master regulator MyoD genome widely acts to alter the epigenome in myoblasts and myotubes. Gagan et al. also performed high-throughput sequencing-based analysis, to find that MyoD binds to the microRNA-378 (miR-378) gene locus and induces transactivation and chromatin remodeling [20]. This activated miRNA directly downregulates the MyoR, a MyoD antagonist, and promotes myogenesis [20].

Genome-wide target gene analyses are also performed in transcription regulators other than the myogenic bHLH factors. Lagha et al. performed ChIP-chip analysis of the transcription factor Pax3 [21], which is essential for ensuring myogenic potential and survival of the progenitors [22]. Pax3 
binds to a sequence $3^{\prime}$ of the Fgfr4 gene that directs Pax3dependent expression at sites of myogenesis in transgenic mouse embryos. The activity of this regulatory element is also partially dependent on E-boxes, targets of the myogenic regulatory factors, which are expressed as progenitor cells entering the myogenic program. Other FGF signaling components, notably Sprouty1, are also regulated by Pax3. These results provide new insight into the Pax-initiated regulatory network that modulates stem cell maintenance versus tissue differentiation. Soleimani et al. performed ChIP-seq analysis of Pax3 and Pax7 [23]. These transcription factors regulate stem cell function in skeletal myogenesis, but little is known about the molecular mechanism of their distinct roles. The genome-wide binding-site analysis combined with gene expression data indicates that both Pax 3 and Pax7 bind identical DNA motifs and jointly activate a large number of genes involved in muscle stem cell function. In adult myoblasts, Pax7 binds to many more sites than the number of genes it regulates. In spite of a significant overlap in their transcriptional network, Pax7 regulates distinct set of genes involved in the acceleration of proliferation and inhibition of myogenic differentiation. Moreover, they showed that Pax7 has a higher binding affinity to the homeodomain-binding motif relative to Pax3, suggesting that the differences in DNA binding contribute to the observed functional difference between Pax3 and Pax7 binding in myogenesis. Mousavi et al. performed ChIP-seq of Polycomb group (PcG) protein Ezh1 and mRNA-seq in skeletal muscle cells [24]. This study provides evidence for genome-wide association of Ezh1 complex with active epigenetic mark (H3K4me3), RNA polymerase II (Pol II), and mRNA production. Although Ezh2, a paralog of Ezh1, is a known trigger for transcription repression by catalyzing the addition of methyl groups onto H3K27 [25], these findings reveal another role for PcG complex in promoting mRNA transcription.

The genome-wide approach also contributes to further understanding of the epigenetic regulation in skeletal myogenesis. Asp et al. examined changes in the chromatin landscape during myogenesis by ChIP-seq analyses of several key histone marks (H3K9Ac, H3K18Ac, H4K12Ac, H2Bub, H3K4me1, H3K4me2, H3K4me3, H3K27me3, and $\mathrm{H} 3 \mathrm{~K} 36 \mathrm{me} 3$ ) and RNA polymerase II in mouse myoblasts and myotubes [26]. Using the data, they identified novel regulatory elements flanking the Myog gene that act as a key differentiation-dependent switch in myogenesis. Myog gene is targeted by PRC2-mediated H3K27 methylation, and its expression is suppressed in myoblasts. Depletion of Suz12, a component of PRC2 complex that regulates H3K27 methylation, led to the loss of PRC2 and H3K27me3 on Myog, resulting in premature and enhanced gene induction. This histone mark could represent part of a methylationacetylation differentiation switch, determining the timing of expression of Myog and therefore terminal differentiation. Vethantham et al. also performed ChIP-seq analyses of $\mathrm{H} 2 \mathrm{Bub}, \mathrm{H} 3 \mathrm{~K} 4 \mathrm{me}$, and H3K79me3 during myogenesis [27]. Ubiquitylation of $\mathrm{H} 2 \mathrm{~B}$ on lysine $120(\mathrm{H} 2 \mathrm{Bub})$ is associated with active transcriptional elongation. H2Bub has been implicated in histone crosstalk and is generally thought to be a prerequisite for trimethylation of $\mathrm{H} 3 \mathrm{~K} 4$ and $\mathrm{H} 3 \mathrm{~K} 79$ in both yeast and mammalian cells. The genome-wide analysis of epigenetic marks identified dynamic loss of H2Bub in the differentiated state. Moreover, they found that the H2B ubiquitin E3 ligase, RNF20, was depleted from chromatin in differentiated myotubes, indicating that recruitment of this protein to genes significantly decreases during myogenesis. Furthermore, they observed retention and gaining of $\mathrm{H} 3 \mathrm{~K} 4$ trimethylation on multiple genes in the absence of H2Bub. The Set1 H3K4 trimethylase complex was efficiently recruited to a subset of genes in myotubes in the absence of $\mathrm{H} 2 \mathrm{Bub}$, suggesting that $\mathrm{H} 3 \mathrm{~K} 4 \mathrm{me} 3$ in the absence of H2Bub in myotubes is mediated via Set1.

Trapnell et al. performed RNA-seq analysis in mouse myoblast cell line representing a differentiation time series [28]. They detected 13,692 known transcripts and 3,724 previously unannotated transcripts. Analysis of transcript expression over the time series revealed complete switches in the dominant transcription start site or splice isoform in 330 genes, along with more subtle changes in further 1,304 genes.

Overall, deep sequencing or array-based approaches have been shown to be of benefit in identifying the molecular network and novel effectors in diverse biological processes. In skeletal myogenesis, these approaches revealed comprehensive target genes of myogenic transcription factors, novel myogenic factors and the characteristics of myoblasts and myotubes, which could not be identified by conventional approaches.

\section{Cell-Based High-Throughput Assay}

Currently, multiple studies have demonstrated comprehensive and cell-based functional screening. Generally, screening for signals activating gene expression consists of examining potential transcription factor-binding sequences in a specific promoter using bioinformatics and reporter assays. If a factor's potential recognition motif is unknown, one-hybrid or South-western screening can be used to identify molecules directly associated with the specific sequence. However, these methods are limited to identifying direct targets only. On the other hand, cell-based reporter assays using a comprehensive set of cDNAs in an expression library allow high-throughput screening not only for direct transcriptional regulators but also for other factors, such as cell-signaling molecules, receptors, and growth factors. Chanda et al. performed a reporter assay-based approach that used about 20,000 annotated cDNAs in the investigation of activator protein-1 (AP-1) signal transduction pathway and identified novel factors of AP-1 mediated growth and mitogenic response pathway [29]. Fiscella et al. performed high-throughput assay using a unique library of cDNAs encoding predicted secreted and transmembrane domain-containing proteins [30]. Supernatants from mammalian cells transiently transfected with this library were incubated with primary $\mathrm{T}$ cells and $\mathrm{T}$ cell lines in several high-throughput assays including reporter and cytokine secretion assay. This identified a T cell factor, TIP ( $\mathrm{T}$ cell immunomodulatory protein), which does not show any homology to proteins with known function. However, treatment of primary human and murine T cells with TIP resulted in the secretion of IFN- $\gamma$, TNF- $\alpha$, and IL-10, whereas 
in vivo TIP had a protective effect in a mouse acute graftversus-host disease (GVHD) model. Konig et al. performed a systematic approach combined with genome-wide siRNA analysis and searched the human interactome database, to uncover multiprotein virus-host interactions that are likely to regulate the early steps of HIV infections [31].

In the myogenesis study, we performed cell-based highthroughput transfection assay to identify activation factors of RP58, a critical myogenesis regulator as described in the latter section.

\section{In Situ Gene Expression Database}

Microarray analysis is a powerful tool to identify the working genes in individual cells or tissues. However, this analysis is unlikely to detect gene expression restricted to small areas. In contrast, in situ hybridization can identify temporal and spatial gene expression patterns. The systematic in situ hybridization database contributes to detailed information for the spatial regulation of gene expression. Gray et al. mapped the expression of 1174 transcription factors in the brain of developing mice using section in situ hybridization [32]. Also, Lein et al. described an anatomically comprehensive digital atlas containing the expression patterns of around 20,000 genes using automated high-throughput procedures for in situ hybridization in the adult mouse brain [33]. These databases describe the anatomical organization of the brain and provide a primary data resource for a wide variety of further studies regarding brain organization and its function.

The Edinburgh Mouse Atlas Gene-Expression Database (EMAGE) is a large-scale database of in situ gene expression patterns of about 16,000 genes in the developing mouse embryo [34-37]. Domains of expression from raw data images are spatially transferred into a set of standard $3 \mathrm{D}$ virtual mouse embryos at different stages of development. Anatomy ontology is also used to describe sites of expression, which allows data to be queried using text-based methods. The GenitoUrinary Development Molecular Anatomy Project (GUDMAP) is also a database of in situ gene expression patterns in mouse embryos [38, 39]. GUDMAP includes whole-mount and section in situ hybridization data of over 3,000 genes and microarray gene expression data of microdissected, laser-captured, and FACS-sorted components of the developing mouse genitourinary (GU) system. These in situ gene expression databases provide more detailed information on the spatial regulation of gene expression and allow identification of discrete clusters of transcribed genes. They serve as a useful source for research in developmental biology.

\section{Our Systems Approach Revealed the MyoD-Mediated Ids Repression Mechanism}

We constructed a unique systems approach and applied it for elucidation of myogenesis molecular network. First, we created our own in situ gene expression database. To identify and characterize effectors of the transcriptional network regulating developmental processes, we developed a web-based comprehensive WISH database for transcriptional regulators using E9.5, 10.5, and 11.5 mouse embryos [13]. We prepared 1520 digoxigenin-labeled RNA probes from cDNA libraries. Using WISH results, we annotated gene expression patterns of each gene and constructed a database, termed "EMBRYS" (http://embrys.jp/embrys/html/MainMenu.html), covering these 3 embryonic days. Using this database, we identified 43 transcription regulators showing myogenic expression pattern in the limb bud. Among those, transcription repressor $R P 58$ was identified as a novel transcription factor expressed in myogenesis [13]. The analysis of RP58 knockout mice revealed that this gene is critical for myogenesis [13]. This database EMBRYS is also useful to identify regulators of another tissue development. Indeed, we also identified that Mohawk homeobox gene is a critical regulator of tendon differentiation by using the database [40].

The WISH database EMBRYS identified a novel transcriptional factor RP58 as a critical regulator of myogenesis. To identify the molecular network anchored by RP58, we investigated the upstream events that promote RP58 expression by cell-based expression vector library transfection assay [13]. We utilized around 6000 arrayed and addressable cDNA clones, which allowed systematic, efficient, and unbiased screening of cDNA encoding factors that could activate the RP58 promoter. A highly conserved RP58 genomic region was inserted in front of luciferase gene in the reporter vector. This was then transfected in 293T cells with expression vector library, and luciferase assay was performed [13]. The highthroughput transfection assay identified myogenic bHLH factor MyoD as a direct transcription activator of RP58 [13].

RP58 has been reported to bind to the specific DNA sequence (A/C)ACATCTG(G/T)(A/C) [41] and is associated with Dnmt3a and Hdac1 [42]. These reports suggest that RP58 can bind to the promoter region of its target genes and repress transcription activity. To identify the repression targets of RP58, we performed microarray analysis and bioinformatics screening by RP58 binding sequence and identified Id 2 and Id 3 as RP58 repression targets [13].

Our systems approach combined with WISH database construction, high-throughput transfection assay, and microarray analysis identified a critical regulatory network of myogenesis (Figure 1). WISH database identified a novel myogenic regulator, RP58. High-throughput transfection screening and microarray analysis identified a MyoDactivated regulatory loop by RP58-mediated repression of myogenic bHLH factor inhibitors Id 2 and Id3. In myoblasts, Ids are expressed and inhibit the myogenic bHLH factors. During myogenesis, RP58 is promoted by MyoD and represses Id transcription. Myogenesis then progresses by myogenic bHLH factor-mediated activation of musclespecific genes (Figure 2). The repression mechanism of Ids had been unclear for almost 20 years, and this new finding indicates the importance of this systems approach.

\section{Conclusion}

A genome-wide systematic approach using high-throughput sequencing technologies, cell-based transfection assays, or 


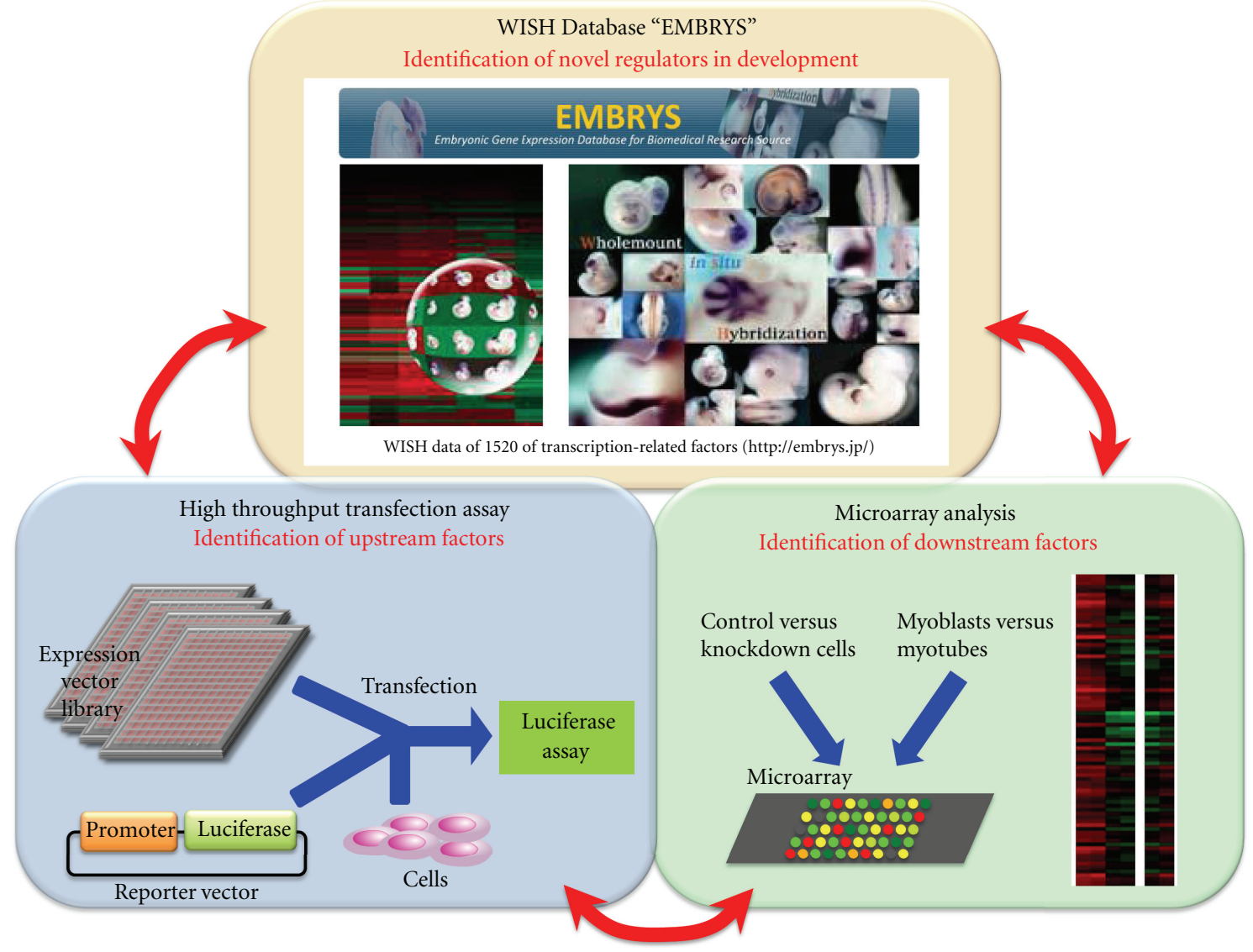

FIGURE 1: Scheme of our systems approach. WISH database EMBRYS identified a novel myogenesis regulator RP58. High-throughput transfection assay and microarray analysis identified upstream and downstream factors of RP58. This multicombined approach is useful for elucidation of molecular network in the developmental process.

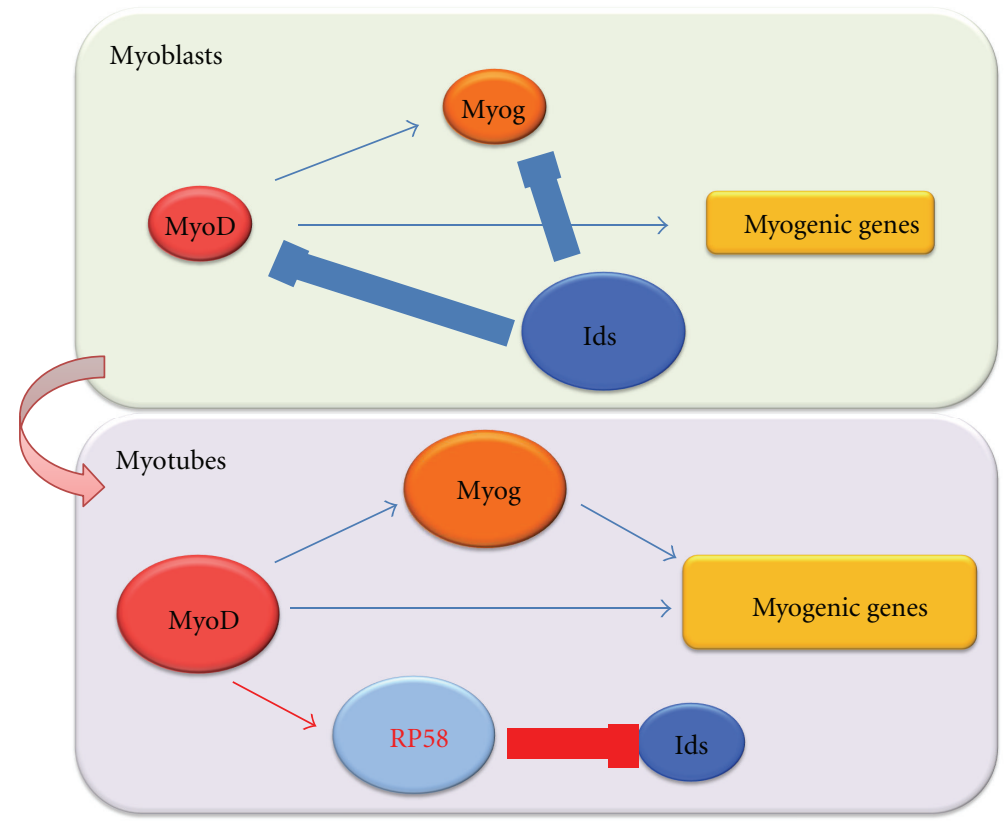

FIGURE 2: Proposed myogenesis regulatory network by our systems approach in myoblasts; Id proteins are expressed and inhibit the myogenic bHLH factors. During myogenic differentiation, RP58 is promoted by MyoD and represses the Id transcription. Muscle specific genes are then activated by myogenic bHLH factors. 
construction of gene expression pattern database is contributing to understanding the mechanisms of various life phenomena. These methods have also been shown to be useful in studying skeletal myogenesis. High-throughput sequencing-based technologies showed genome-wide target genes of myogenesis regulators and epigenetic modification in skeletal myogenesis. We also identified a novel myogenesis network regulated by RP58 using the multicombined approach. Although the myogenesis study using systems approach is still at its early stages, the systems approach will enable further understanding of myogenesis in the future.

\section{References}

[1] A. Blais, M. Tsikitis, D. Acosta-Alvear, R. Sharan, Y. Kluger, and B. D. Dynlacht, "An initial blueprint for myogenic differentiation," Genes \& Development, vol. 19, pp. 553-569, 2005.

[2] Y. Cao, R. M. Kumar, B. H. Penn et al., "Global and genespecific analyses show distinct roles for Myod and Myog at a common set of promoters," The EMBO Journal, vol. 25, pp. 502-511, 2006.

[3] B. H. Penn, D. A. Bergstrom, F. J. Dilworth, E. Bengal, and S. J. Tapscott, "A MyoD -generated feed-forward circuit temporally patterns gene expression dining skeletal muscle differentiation," Genes and Development, vol. 18, no. 19, pp. 2348-2353, 2004.

[4] M. A. Rudnicki, P. N. J. Schnegelsberg, R. H. Stead, T. Braun, H. H. Arnold, and R. Jaenisch, "MyoD or Myf-5 is required for the formation of skeletal muscle," Cell, vol. 75, no. 7, pp. 1351-1359, 1993.

[5] Y. Nabeshima, K. Hanaoka, M. Hayasaka et al., "Myogeningene disruption results in perinatal lethality because of severe muscle defect," Nature, vol. 364, pp. 532-535, 1993.

[6] P. Hasty, A. Bradley, J. H. Morris et al., "Muscle deficiency and neonatal death in mice with a targeted mutation in the myogenin gene," Nature, vol. 364, pp. 501-506, 1993.

[7] L. Kassar-Duchossoy, B. Gayraud-Morel, D. Gomès et al., "Mrf4 determines skeletal muscle identity in Myf5: myod double-mutant mice," Nature, vol. 431, no. 7007, pp. 466-471, 2004.

[8] C. A. Berkes and S. J. Tapscott, "MyoD and the transcriptional control of myogenesis," Seminars in Cell \& Developmental Biology, vol. 16, no. 4-5, pp. 585-595, 2005.

[9] B. Chen, B. H. Han, X. H. Sun, and R. W. Lim, "Inhibition of muscle-specific gene expression by Id3: requirement of the C-terminal region of the protein for stable expression and function," Nucleic Acids Res, vol. 25, no. 2, pp. 423-430, 1997.

[10] K. Langlands, X. Yin, G. Anand, and E. V. Prochownik, "Differential interactions of Id proteins with basic-helix-loophelix transcription factors," Journal of Biological Chemistry, vol. 272, no. 32, pp. 19785-19793, 1997.

[11] I. N. Melnikova, M. Bounpheng, G. C. Schatteman, D. Gilliam, and B. A. Christy, "Differential biological activities of mammalian Id proteins in muscle cells," Experimental Cell Research, vol. 247, no. 1, pp. 94-104, 1999.

[12] R. Benezra, R. L. Davis, D. Lockshon, D. L. Turner, and H. Weintraub, "The protein Id: a negative regulator of helix-loophelix DNA binding proteins," Cell, vol. 61, no. 1, pp. 49-59, 1990.

[13] S. Yokoyama, Y. Ito, H. Ueno-Kudoh et al., "A systems approach reveals that the myogenesis genome network is regulated by the transcriptional repressor RP58," Developmental Cell, vol. 17, no. 6, pp. 836-848, 2009.

[14] A. Meissner, T. S. lsen, H. Gu et al., "Genome-scale DNA methylation maps of pluripotent and differentiated cells," Nature, vol. 454, pp. 766-770, 2008.

[15] C. Bock, E. Kiskinis, G. Verstappen et al., "Reference maps of human ES and iPS cell variation enable high-throughput characterization of pluripotent cell lines," Cell, vol. 144, no. 3 , pp. 439-452, 2011.

[16] B. E. Bernstein, T. S. Mikkelsen, X. Xie et al., "A bivalent chromatin structure marks key developmental genes in embryonic stem cells," Cell, vol. 125, no. 2, pp. 315-326, 2006.

[17] G. Pan, S. Tian, J. Nie et al., "Whole-genome analysis of histone $\mathrm{H} 3$ lysine 4 and lysine 27 methylation in human embryonic stem cells," Cell Stem Cell, vol. 1, no. 3, pp. 299312, 2007.

[18] J. M. Calabrese, A. C. Seila, G. W. Yeo, and P. A. Sharp, "RNA sequence analysis defines Dicer's role in mouse embryonic stem cells," Proceedings of the National Academy of Sciences of the USA, vol. 104, no. 46, pp. 18097-18102, 2007.

[19] Y. Cao, Z. Yao, D. Sarkar et al., "Genome-wide MyoD binding in skeletal muscle cells: a potential for broad cellular reprogramming," Developmental Cell, vol. 18, no. 4, pp. 662674, 2010.

[20] J. Gagan, B. K. Dey, R. Layer, Z. Yan, and A. Dutta, "MicroRNA-378 targets the myogenic repressor MyoR during myoblast differentiation," Journal of Biological Chemistry, vol. 286, no. 22, pp. 19431-19438, 2011.

[21] M. Lagha, J. D. Kormish, D. Rocancourt et al., "Pax3 regulation of FGF signaling affects the progression of embryonic progenitor cells into the myogenic program," Genes \& Development, vol. 22, pp. 1828-1837, 2008.

[22] M. Buckingham and F. Relaix, "The role of pax genes in the development of tissues and organs: Pax3 and Pax7 regulate muscle progenitor cell functions," Annual Review of Cell and Developmental Biology, vol. 23, pp. 645-673, 2007.

[23] V. D. Soleimani, V. G. Punch, Y. Kawabe et al., "Transcriptional dominance of Pax7 in adult Myogenesis is due to high-affinity recognition of homeodomain motifs," Developmental Cell, vol. 22, no. 6, pp. 1208-1220, 2012.

[24] K. Mousavi, H. Zare, A. H. Wang, and V. Sartorelli, "Polycomb protein Ezh1 promotes RNA polymerase II elongation," Molecular Cell, vol. 45, no. 2, pp. 255-262, 2012.

[25] J. A. Simon and R. E. Kingston, "Mechanisms of polycomb gene silencing: knowns and unknowns," Nature Reviews Molecular Cell Biology, vol. 10, pp. 697-708, 2009.

[26] P. Asp, R. Bluma, V. Vethanthama et al., "Genome-wide remodeling of the epigenetic landscape during myogenic differentiation," Proceedings of the National Academy of Sciences of the USA, vol. 108, no. 22, pp. E149-E158, 2011.

[27] V. Vethantham, Y. Yanga, C. Bowmana et al., "Dynamic loss of $\mathrm{H}_{2} \mathrm{~B}$ ubiquitylation without corresponding changes in $\mathrm{H}_{3} \mathrm{~K}_{4}$ trimethylation during myogenic differentiation," Molecular and Cellular Biology, vol. 32, no. 6, pp. 1044-1055, 2012.

[28] C. Trapnell, B. A. Williams, G. Pertea et al., "Transcript assembly and quantification by RNA-Seq reveals unannotated transcripts and isoform switching during cell differentiation," Nature Biotechnology, vol. 28, pp. 511-515, 2010.

[29] S. K. Chanda, S. White, A. P. Orth et al., "Genome-scale functional profiling of the mammalian AP-1 signaling pathway," Proceedings of the National Academy of Sciences of USA, vol. 100, pp. 12153-12158, 2003.

[30] M. Fiscella, J. W. Perry, B. Teng et al., "TIP, a T-cell factor identified using high-throughput screening increases survival 
in a graft-versus-host disease model," Nature Biotechnology, vol. 21, pp. 302-307, 2003.

[31] R. Konig, Y. Zhou, D. Elleder et al., "Global analysis of host-pathogen interactions that regulate early-stage HIV-1 replication," Cell, vol. 135, no. 1, pp. 49-60, 2008.

[32] P. A. Gray, H. Fu, P. Luo et al., "Mouse brain organization revealed through direct genome-scale TF expression analysis," Science, vol. 306, no. 5705, pp. 2255-2257, 2004.

[33] E. S. Lein, M. J. Hawrylycz, N. Ao et al., "Genome-wide atlas of gene expression in the adult mouse brain," Nature, vol. 445, pp. 168-176, 2007.

[34] R. A. Baldock, J. B. L. Bard, A. Burger et al., "EMAP and EMAGE: a framework for understanding spatially organized data," Neuroinformatics, vol. 1, no. 4, pp. 309-325, 2003.

[35] L. Richardson, S. Venkataraman, P. Stevenson et al., "EMAGE mouse embryo spatial gene expression database: 2010 update," Nucleic Acids Research, vol. 38, supplement 1, pp. D703-D709, 2010.

[36] S. Venkataraman, P. Stevenson, Y. Yang et al., "EMAGEedinburgh mouse atlas of gene expression: 2008 update," Nucleic Acids Research, vol. 36, supplement 1, pp. D860-D865, 2008.

[37] J. H. Christiansen, Y. Yang, S. Venkataraman et al., "EMAGE: a spatial database of gene expression patterns during mouse embryo development," Nucleic Acids Research, vol. 34, no. 1, pp. D637-D641, 2006.

[38] S. D. Harding, C. Armit, J. Armstrong et al., "The GUDMAP database-an online resource for genitourinary research," Development, vol. 138, pp. 2845-2853, 2011.

[39] A. P. McMahon, B. J. Aronow, D. R. Davidson et al., "GUDMAP: the genitourinary developmental molecular anatomy project," Journal of the American Society of Nephrology, vol. 19, no. 4, pp. 667-671, 2008.

[40] Y. Ito, N. Toriuchia, T. Yoshitakaa et al., "The Mohawk homeobox gene is a critical regulator of tendon differentiation," Proceedings of the National Academy of Sciences of USA, vol. 107, no. 23, pp. 10538-10542, 2010.

[41] K. Aoki, G. Meng, K. Suzuki et al., "RP58 associates with condensed chromatin and mediates a sequence-specific transcriptional repression," The Journal of Biological Chemistry, vol. 273, pp. 26698-26704, 1998.

[42] F. Fuks, W. A. Burgers, N. Godin, M. Kasai, and T. Kouzarides, "Dnmt3a binds deacetylases and is recruited by a sequencespecific repressor to silence transcription," EMBO Journal, vol. 20, no. 10, pp. 2536-2544, 2001. 

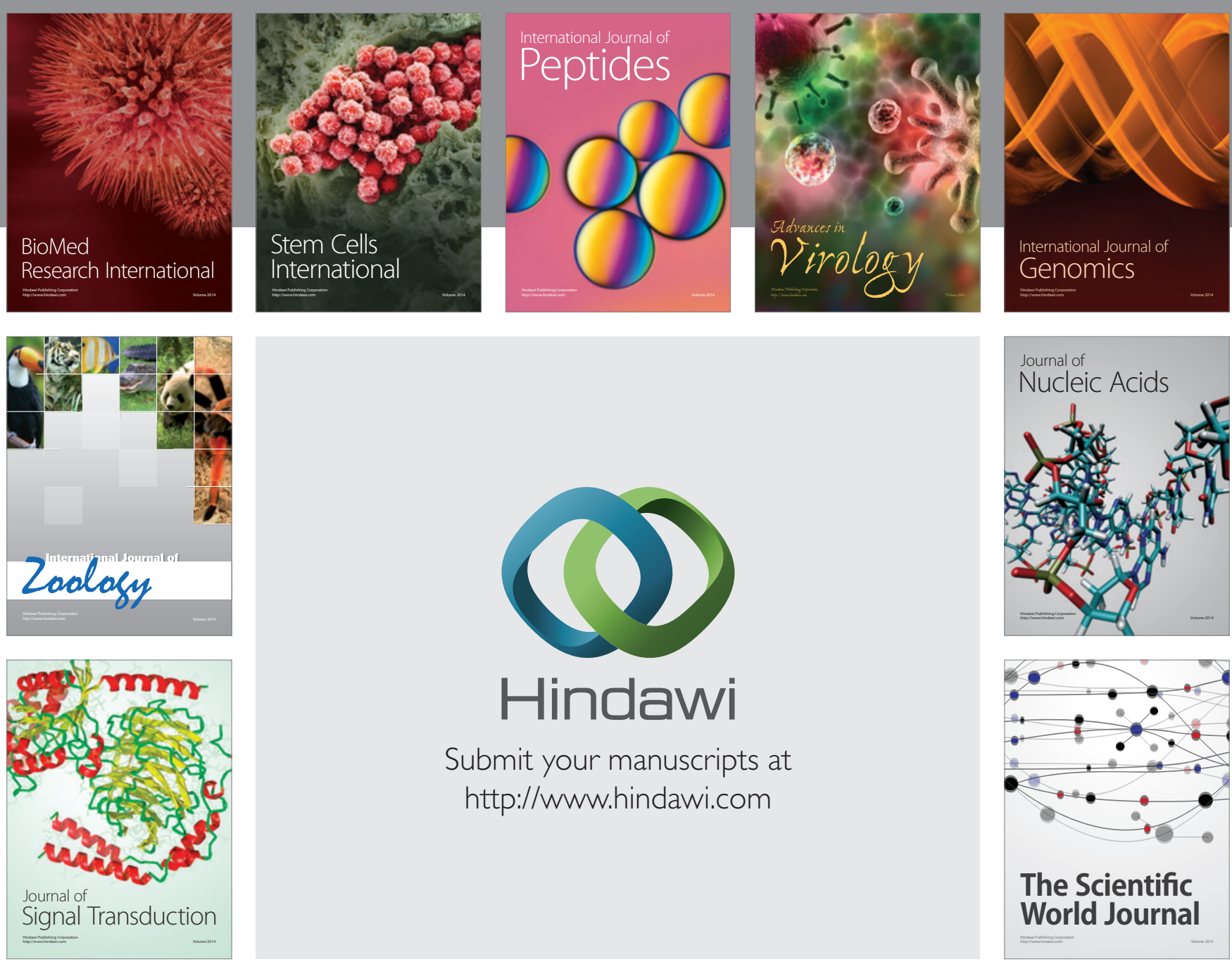

Submit your manuscripts at

http://www.hindawi.com
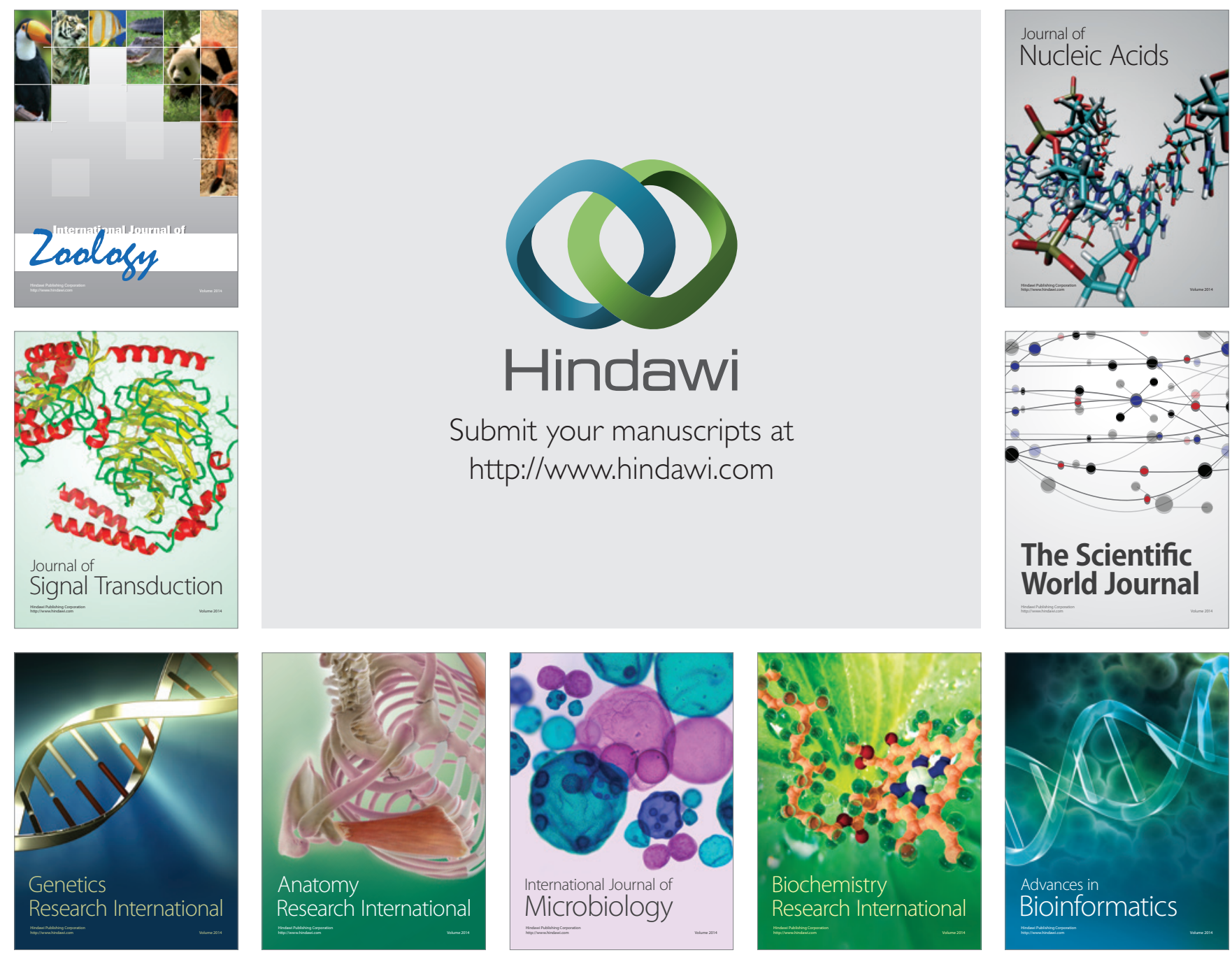

The Scientific World Journal
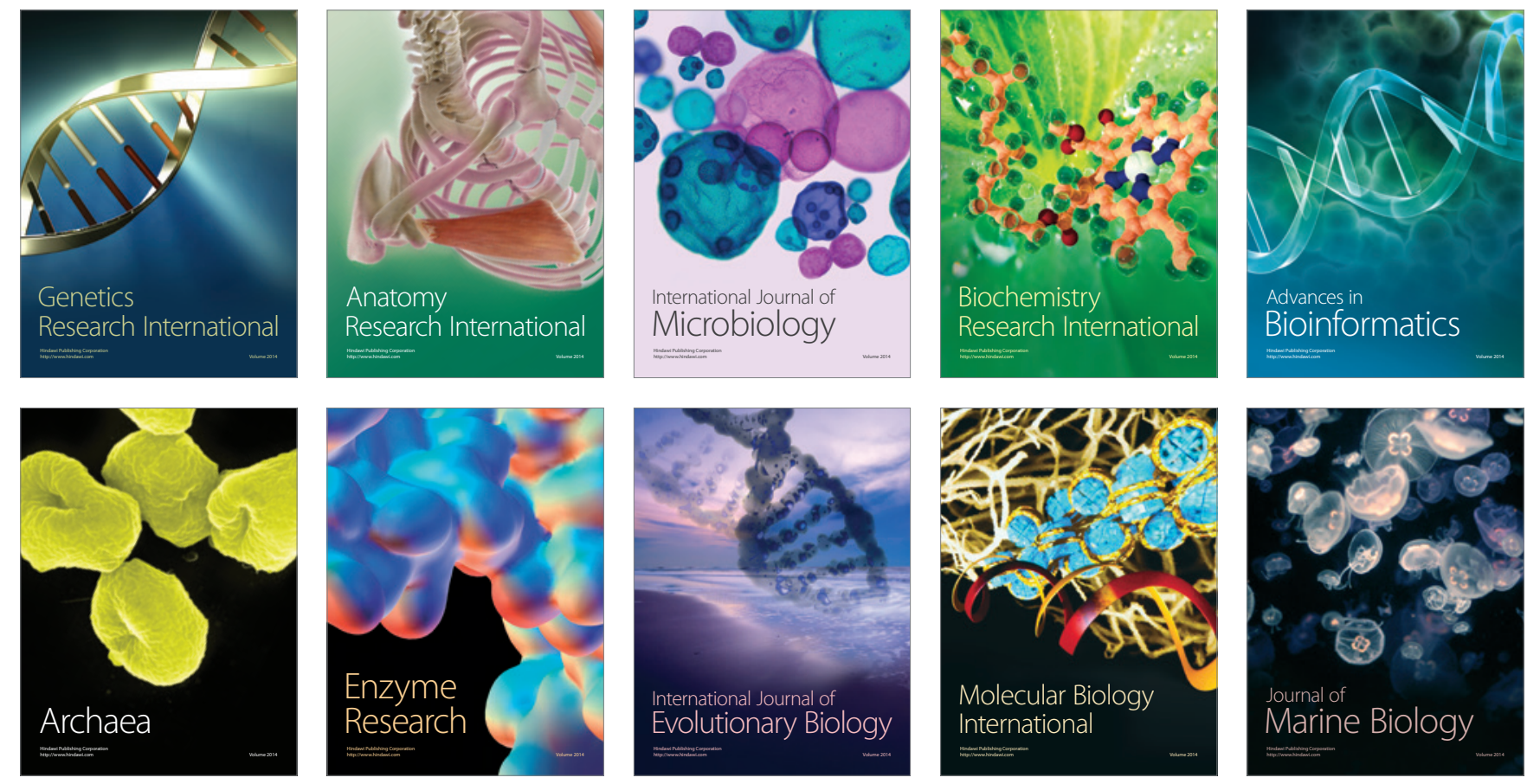